\title{
Extension of the spectral element method for stability analysis of time-periodic delay-differential equations with multiple and distributed delays
}

\author{
David Lehotzky*, a, Tamas Insperger ${ }^{\mathrm{a}}$, and Gabor Stepan ${ }^{\mathrm{a}}$ \\ ${ }^{a}$ Department of Applied Mechanics, Budapest University of Technology and Economics, Hungary, \\ 1111, Budapest, Muegyetem rkp. 3.
}

\begin{abstract}
The spectral element method was introduced by Khasawneh and Mann (2013) for the stability analysis of time-periodic delay-differential equations (DDEs) with multiple delays. In this paper, this method is generalized for time-periodic DDEs with multiple delays and distributed delay. For this general case, an explicit formula is given for the construction of the matrix approximation of the monodromy operator. The derived formula enables the algorithmic application of the method to DDEs with general combinations of delays for arbitrary point sets and test functions. Stability analysis is demonstrated for specific case studies, and the computation code is provided for a complex example.
\end{abstract}

Keywords: time-delay system; time-periodic system; stability; spectral element

${ }^{*}$ Corresponding author

E-mail address: lehotzky@mm.bme.hu 


\section{Introduction}

Time-delay systems are often used for mathematical modelling in engineering and biological sciences. Models involving delayed terms can be found in the literature of machining operations [2], feedback control systems [3], wheel dynamics [29], traffic dynamics [24], human balancing [23, 28], population dynamics [21] and epidemiology [25], just to mention a few areas of application. Although time dependency of system parameters is often neglected in these models, this simplification cannot be justified in all cases. For instance, in milling operations [27], in turning operations with spindle speed variation (see Chapter 5.1.3 in [17]) and in digitally controlled systems [13] time-dependent parameters play essential role and affect stability properties. Note that time-periodic systems often arise in the mathematical models of engineering applications [9, 26]. For the majority of the applications of dynamical systems, it is desired to keep the system in the proximity of a particular state or motion by the proper tuning of system and control parameters. Therefore, increasing attention has been given to the stability properties of time-delay systems and, as a result, many analytical and numerical methods can be found on their linear stability analysis. In the recent decades several numerical methods have been developed for the linear stability analysis of time-periodic delay-differential equations (DDEs). Examples for such methods are the multifrequency solution [1, 5], semi-discretization [17], full discretization [12], complete discretization [22], Chebyshev continuous time approximation [10] and the recently presented spectral element method [18, 19, 20], which is the subject of this paper. The method was first published in 18 for autonomous DDEs with single delay, thereafter it was extended in [19] to autonomous DDEs with distributed delay and generalized to time-periodic DDEs with multiple point delays in [20]. Applications to delayed feedback control of chaos were presented in [31, 32].

The general form of linear, time-periodic DDEs is

$$
\dot{\mathbf{x}}(t)=\mathcal{L}(t) \mathbf{x}^{t-\tau, t}, \quad t \geq 0
$$

where $\mathcal{L}(t)=\mathcal{L}(t+T), \forall t$ is a linear, time-periodic functional, with $T$ being the principal period. Here and from now on, notation $\mathbf{x}^{a, b}$ is applied for solution segment $\{\mathbf{x}(\theta): \theta \in[a, b]\}$, thus in (1), solution segment $\mathbf{x}^{t-\tau, t}=\{\mathbf{x}(\theta): \theta \in[t-\tau, t]\}$ contains the history of the state dating back to $\tau$. The stability of $(1)$ is determined by the monodromy operator $\mathcal{U}(T)$, which maps the initial solution segment $\mathbf{x}^{-\tau, 0}$ to $\mathbf{x}^{T-\tau, T}$. System (1) is stable if and only if all characteristic multipliers (non-zero eigenvalues) of $\mathcal{U}(T)$ are within the unit circle of the complex plane (for details see Chapter 8 in [14]).

In [20], the spectral element method gives a matrix approximation $\mathbf{U}$ of the monodromy operator for DDEs with point delays, that is for the case when

$$
\begin{aligned}
\mathcal{L}(t) \mathbf{x}^{t-\tau, t} & =\mathbf{A}(t) \int_{-\tau}^{0} \delta(\theta) \mathbf{x}(t+\theta) \mathrm{d} \theta+\sum_{l=1}^{u} \mathbf{B}_{l}(t) \int_{-\tau}^{0} \delta\left(\theta+\tau_{l}\right) \mathbf{x}(t+\theta) \mathrm{d} \theta \\
& =\mathbf{A}(t) \mathbf{x}(t)+\sum_{l=1}^{u} \mathbf{B}_{l}(t) \mathbf{x}\left(t-\tau_{l}\right)
\end{aligned}
$$

with $\delta(\theta)$ being the Dirac delta function, $\mathbf{x}: \mathbb{R} \rightarrow \mathbb{R}^{s} ; \mathbf{A}, \mathbf{B}_{l}: \mathbb{R} \rightarrow \mathbb{R}^{s \times s}, 0<\tau_{l}, l=1,2, \ldots, u$; $\tau \geq \max \left\{\tau_{l}\right\}_{l=1}^{u}$ and $\mathbf{A}(t)=\mathbf{A}(t+T) ; \mathbf{B}_{l}(t)=\mathbf{B}_{l}(t+T), l=1,2, \ldots, u ; \forall t$. Under increasing degree of the approximation scheme the matrix approximation $\mathbf{U}$ provides convergent results for the stability of the original system, given by (1) and (2). This property of the spectral element method is shown in [20] for several numerical examples. Although the spectral element method seems to be a handy tool for the stability analysis of time-periodic DDEs, its application is not straightforward due to the lack of explicit formulas for the computation of $\mathbf{U}$. While explicit formulas were presented in [18] for the construction of $\mathbf{U}$ for autonomous DDEs with 
a single point delay, [19] and [20] does not provide any explicit formula for the general case with multiple point and distributed delays and with time-periodic coefficients. This paper gives explicit formulas for all components of the matrices, which are necessary for the computation of $\mathbf{U}$. In contrast with [20], here the formula of $\mathbf{U}$ is derived using operator equations. Results are presented in the form of stability charts for particular time-periodic DDEs.

The structure of the paper is the following. First, the derivation of the method is presented for a general type of time-periodic DDE, which involves point delays and one term of distributed delay. Thereafter the method is applied to particular systems. Finally, some conclusions are given.

\section{Derivation of the method}

In this section the derivation of the spectral element method is presented for a DDE with multiple point delays and one distributed-delay term. The investigated linear time-periodic DDE reads

$$
\dot{\mathbf{x}}(t)=\mathbf{A}(t) \mathbf{x}(t)+\sum_{l=1}^{u} \mathbf{B}_{l}(t) \mathbf{x}\left(t-\tau_{l}\right)+\int_{-a}^{-b} \gamma(t, \theta) \mathbf{x}(t+\theta) \mathrm{d} \theta,
$$

where $0 \leq b<a ; \gamma: \mathbb{R}^{2} \rightarrow \mathbb{R}^{s \times s} ;$ and $\gamma(t, \theta)=\gamma(t+T, \theta), \forall \theta, \forall t$. With the application of numerical integration using Lobatto-type Gaussian quadrature, (3) can be approximated by the time-periodic DDE

$$
\dot{\mathbf{x}}(t)=\mathbf{A}(t) \mathbf{x}(t)+\sum_{p=1}^{v} \mathbf{B}_{p}(t) \mathbf{x}\left(t-\tau_{p}\right)
$$

where

$$
\mathbf{B}_{p}(t)=\frac{a-b}{2} \gamma\left(t, \theta_{p-u}\right) w_{p-u}
$$

$\tau_{p}=-\theta_{p-u}$ for $p=u+1, u+2, \ldots, v$, while $v=u+m$ and $\theta_{q}=\frac{a-b}{2} \eta_{q}-\frac{a+b}{2} \in[-a,-b]$, with $\left\{\eta_{q}\right\}_{q=1}^{m} \subset[-1,1]$ and $\left\{w_{q}\right\}_{q=1}^{m}$ being the set of Lobatto-type Gaussian quadrature nodes and weights, respectively (see Appendix C for details). Note that quadratures other than the Lobatto-type Gaussian quadrature can also be used for integration. In fact, the standard Gaussian quadrature or the Clenshaw-Curtis quadrature can increase the order of accuracy of the approximate system (4) (see [30] for details). Here the Lobatto-type Gaussian quadrature is used because it still keeps a good accuracy and the proposed numerical method reuses it during the calculation. Note that (4) contains point delays only, hence it can be concluded that DDEs with point delays can approximate the case when terms with distributed delays are also present. In the following, the approximate system (4) is studied and $\tau_{1} \leq \tau_{2} \leq \ldots \leq \tau_{v}$ is assumed for convenience. Consider the solution segment $\mathbf{x}^{-\tau, T}$, where $\tau=K T$ is the monitored history, with

$$
K= \begin{cases}\operatorname{int}\left(\tau_{v} / T\right) & \text { if } \tau_{v} \bmod T=0 \\ \operatorname{int}\left(\tau_{v} / T\right)+1 & \text { otherwise }\end{cases}
$$

while int $(\cdot)$ denotes the integer part function. The approximate system (4) is equivalent to the operator equation

$$
\mathcal{A} \mathbf{x}^{-\tau, T}=\mathbf{0}
$$

where the linear operator $\mathcal{A}$ is defined by

$$
\mathcal{A} \mathbf{x}^{-\tau, T}=\left\{\dot{\mathbf{x}}(t)-\mathbf{A}(t) \mathbf{x}(t)-\sum_{p=1}^{v} \mathbf{B}_{p}(t) \mathbf{x}\left(t-\tau_{p}\right): t \in[0, T]\right\} .
$$




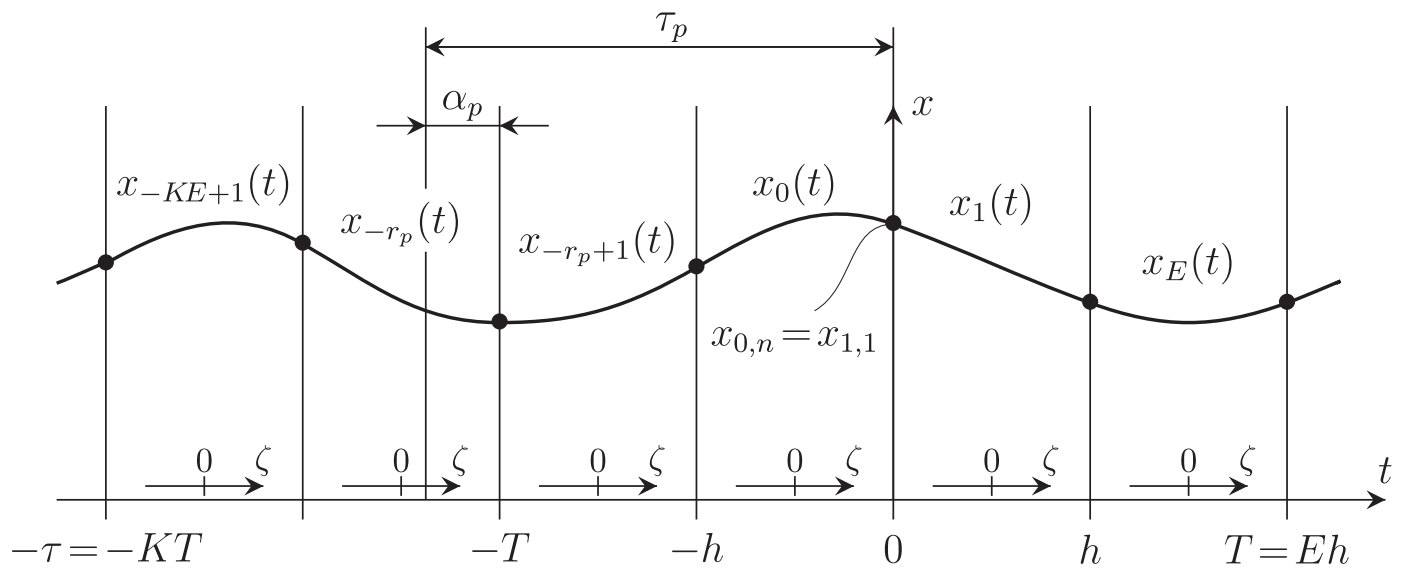

Figure 1: Splitting of the solution segment $\mathbf{x}^{-\tau, T}$ for the case $s=1, K=2$ and $E=2$. The depicted delay $\tau_{p}$ results in $r_{p}=2$.

Now split the solution segment $\mathbf{x}^{-\tau, T}$ onto $(K+1) E$ number of equidistant sub-segments (referred to as elements in the following) as

$$
\mathbf{x}_{k}=\mathbf{x}^{(k-1) h, k h}, \quad k=-E K+1,-E K+2, \ldots, E ;
$$

where $h=T / E$ denotes the length of elements. The splitting of solution segment $\mathbf{x}^{-\tau, T}$ is illustrated in Figure 1 for specific $s, K$ and $E$ parameters. Subsequent elements are connected at their boundaries, therefore conditions

$$
\mathbf{x}_{k}(k h)=\mathbf{x}_{k+1}(k h), \quad k=-E K+1,-E K+2, \ldots, E-1
$$

are valid. The splitting of solution segment $\mathbf{x}^{-\tau, T}$ transforms (4) to a system of differential equations of the form

$$
\dot{\mathbf{x}}_{k}(t)=\mathbf{A}(t) \mathbf{x}_{k}(t)+\sum_{p=1}^{v} \mathbf{B}_{p}(t) \mathbf{x}_{k, p}^{*}(t), \quad t \in((k-1) h, k h], k=1,2, \ldots, E ;
$$

with boundary conditions 10 and

$$
\mathbf{x}_{k, p}^{*}(t)= \begin{cases}\mathbf{x}_{k-r_{p}-1}\left(t-\tau_{p}\right) & \text { if }\left(t-\tau_{p}\right) \in\left(\left(k-r_{p}-2\right) h,\left(k-r_{p}-1\right) h\right] \\ \mathbf{x}_{k-r_{p}}\left(t-\tau_{p}\right) & \text { if }\left(t-\tau_{p}\right) \in\left(\left(k-r_{p}-1\right) h,\left(k-r_{p}\right) h\right],\end{cases}
$$

where $r_{p}=\operatorname{int}\left(\tau_{p} / h\right)$ (see the illustration in Figure 1). System (11) of differential equations are equivalent to the system of operator equations

$$
\mathcal{S}^{k} \mathbf{x}_{k}-\sum_{p=1}^{v} \mathcal{Q}^{k, p} \mathbf{x}_{k-r_{p}-1}-\sum_{p=1}^{v} \boldsymbol{\mathcal { R }}^{k, p} \mathbf{x}_{k-r_{p}}=\mathbf{0}, \quad k=1,2, \ldots, E ;
$$

where the operators are defined as

$$
\begin{aligned}
\mathcal{S}^{k} \mathbf{x}_{k} & = \begin{cases}\dot{\mathbf{x}}_{k}(t)-\mathbf{A}(t) \mathbf{x}_{k}(t) & \text { if } t \in((k-1) h, k h], \\
\mathbf{0} & \text { otherwise },\end{cases} \\
\mathcal{Q}^{k, p} \mathbf{x}_{k-r_{p}-1} & = \begin{cases}\mathbf{B}_{p}(t) \mathbf{x}_{k-r_{p}-1}\left(t-\tau_{p}\right) & \text { if }\left(t-\tau_{p}\right) \in\left(\left(k-r_{p}-1\right) h-\alpha_{p},\left(k-r_{p}-1\right) h\right], \\
\mathbf{0} & \text { otherwise, }\end{cases} \\
\boldsymbol{\mathcal { R }}^{k, p} \mathbf{x}_{k-r_{p}} & = \begin{cases}\mathbf{B}_{p}(t) \mathbf{x}_{k-r_{p}}\left(t-\tau_{p}\right) & \text { if }\left(t-\tau_{p}\right) \in\left(\left(k-r_{p}-1\right) h,\left(k-r_{p}\right) h-\alpha_{p}\right], \\
\mathbf{0} & \text { otherwise, }\end{cases}
\end{aligned}
$$


with $\alpha_{p}=\tau_{p} \bmod h$ (see the illustration in Figure 1). By the introduction of element-wise (local) coordinates

$$
\zeta_{k}=\frac{2(t-(k-1) h)}{h}-1, \quad k=-E K+1,-E K+2, \ldots, E
$$

and dropping the index $k$ immediately (see the illustration in Figure 1), operators (14) $-(16)$ assume the form

$$
\begin{aligned}
\mathcal{S}^{k} \mathbf{x}_{k} & = \begin{cases}\frac{2}{h} \mathbf{x}_{k}^{\prime}(\zeta)-\mathbf{A}\left(\frac{h(\zeta+1)}{2}+(k-1) h\right) \mathbf{x}_{k}(\zeta) & \text { if } \zeta \in(-1,1], \\
\mathbf{0} & \text { otherwise, }\end{cases} \\
\mathcal{Q}^{k, p} \mathbf{x}_{k-r_{p}-1} & = \begin{cases}\mathbf{B}_{p}\left(\frac{h(\zeta+1)}{2}+(k-1) h\right) \mathbf{x}_{k-r_{p}-1}\left(\zeta+2-\beta_{p}\right) & \text { if } \zeta \in\left(-1,-1+\beta_{p}\right], \\
\mathbf{0} & \text { otherwise, }\end{cases} \\
\mathcal{R}^{k, p} \mathbf{x}_{k-r_{p}} & = \begin{cases}\mathbf{B}_{p}\left(\frac{h(\zeta+1)}{2}+(k-1) h\right) \mathbf{x}_{k-r_{p}}\left(\zeta-\beta_{p}\right) & \text { if } \zeta \in\left(-1+\beta_{p}, 1\right], \\
\mathbf{0} & \text { otherwise, }\end{cases}
\end{aligned}
$$

where $\beta_{p}=2 \alpha_{p} / h$. The application of element-wise coordinate transformation (17) to boundary conditions 10$)$ gives

$$
\mathbf{x}_{k}(1)=\mathbf{x}_{k+1}(-1), \quad k=-E K+1,-E K+2, \ldots, E-1 .
$$

The splitting of solution segment $\mathrm{x}^{-\tau, T}$ thus transformed operator equation (7) to a system (13) of operator equations subject to boundary conditions (21).

Operator equations (13) can be converted into their weak forms after taking their scalar product with test function $\psi$, which gives

$$
\left\langle\mathcal{S}^{k} \mathbf{x}_{k}, \psi\right\rangle-\sum_{p=1}^{v}\left\langle\mathcal{Q}^{k, p} \mathbf{x}_{k-r_{p}-1}, \psi\right\rangle-\sum_{p=1}^{v}\left\langle\boldsymbol{\mathcal { R }}^{k, p} \mathbf{x}_{k-r_{p}}, \psi\right\rangle=\mathbf{0}, \quad k=1,2, \ldots, E .
$$

Here the scalar product of some functions $f$ and $g$ is defined according to

$$
\langle f, g\rangle=\int_{a}^{b} f(\zeta) g(\zeta) \mathrm{d} \zeta
$$

where $[a, b]$ is the domain of functions $f$ and $g$. The solution of (13) always satisfies (22) however, if a solution satisfies (22), that does not imply that it also satisfies (13). System of equations (13) and (22) are equivalent if and only if (22) is true for all possible $\psi$ test functions of the function space (i.e., if the left-hand side of (13) is orthogonal to all elements of the function space). Using $\psi$ with this purport, 22 is called the weak form of (13).

The spectral element method approximates each element $\mathbf{x}_{k}$ with its Lagrange interpolant as

$$
\tilde{\mathbf{x}}_{k}(\zeta)=\sum_{j=1}^{n} \phi_{j}(\zeta) \mathbf{x}_{k, j}, \quad k=-E K+1,-E K+2, \ldots, E
$$

where $\mathbf{x}_{k, j}=\mathbf{x}_{k}\left(\zeta_{j}\right)$ and $\phi_{j}(\zeta)$ are the Lagrange base polynomials (for details on Lagrange interpolation see Appendix A. The node set of interpolation $\left\{\zeta_{j}\right\}_{j=1}^{n} \subset[-1,1]$ is chosen to be of Lobatto-type, that is there are nodes on the endpoints of interval $\zeta \in[-1,1]$, which simplifies boundary conditions (21) to

$$
\mathbf{x}_{k, n}=\mathbf{x}_{k+1,1}, \quad k=-E K+1,-E K+2, \ldots, E-1
$$


(see the illustration in Figure 1 for $k=0$ ). Note that together with boundary conditions (25), (24) gives a piecewise Lagrange interpolant for $\mathbf{x}^{-\tau, T}$. After the substitution of (24) to (22), one obtains

$$
\left\langle\mathbf{r}_{k}, \psi\right\rangle=\mathbf{0}, \quad k=1,2, \ldots, E
$$

where

$$
\mathbf{r}_{k}=\mathcal{S}^{k} \tilde{\mathbf{x}}_{k}-\sum_{p=1}^{v} \mathcal{Q}^{k, p} \tilde{\mathbf{x}}_{k-r_{p}-1}-\sum_{p=1}^{v} \boldsymbol{\mathcal { R }}^{k, p} \tilde{\mathbf{x}}_{k-r_{p}} \neq \mathbf{0}, \quad k=1,2, \ldots, E ;
$$

are the residual functions. Since the approximate solution (24) is an element of the space of $(n-1)$ - degree polynomials (that is $\tilde{\mathbf{x}}_{k} \in \mathcal{P}_{n-1}$ ), the orthogonality of $\mathbf{r}_{k}$ to all possible elements of the subspace of approximation can be ensured by setting $\mathbf{r}_{k}$ orthogonal to a basin $\left\{\psi_{i}\right\}_{i=1}^{n}$ of $\mathcal{P}_{n-1}$, i.e.

$$
\left\langle\mathbf{r}_{k}, \psi_{i}\right\rangle=\mathbf{0}, \quad i=1,2, \ldots, n ; k=1,2, \ldots, E .
$$

This system of equations gives the finite dimensional approximation of weak form (22). Since (28) weights $\mathbf{r}_{k}$ residual functions by $\psi_{i}$ test functions along the domain of solution, this method, used for the discretization of operator equations, was named weighted residuals by Crandall in [11. In order to avoid the accumulation of rounding errors let us choose an orthogonal basin for $\left\{\psi_{i}\right\}_{i=1}^{n}$, given by Legendre polynomials with degree up to $n-1$, that is $\psi_{i}=P_{i-1} ; i=1,2, \ldots, n$; where $P_{i}$ is a Legendre polynomial with degree $i$ (for details on Legendre polynomials see Appendix B $)$. Note that (28) defines $E n$ number of linear algebraic equations. For a given initial function these equations contain $E n$ number of unknown parameters $\left(\mathbf{x}_{k, j} ; k=1,2, \ldots, E ; j=\right.$ $1,2, \ldots, n)$, however, there are further constraints for $\tilde{\mathbf{x}}_{k}$ solution segments, given by boundary conditions (25). These boundary conditions provide $E$ number of additional equations, which have to be satisfied by solution segments $\tilde{\mathbf{x}}_{k}$. In [20], the equations corresponding to $\psi_{n}$ in (28), were replaced by the boundary conditions with running index $k=0,1, \ldots, E-1$ in (25). This was done, in order to obtain the same number of equations as variables. This approach gives the system

$$
\left\langle\mathbf{r}_{k}, \psi_{i}\right\rangle=\mathbf{0}, \quad i=1,2, \ldots, n-1 ; k=1,2, \ldots, E
$$

of linear algebraic equations with unknown parameters $\mathbf{x}_{k, j} ; k=1,2, \ldots, E ; j=2,3, \ldots, n$. The left-hand-side in 29 can be expanded as

$$
\begin{aligned}
\left\langle\mathbf{r}_{k}, \psi_{i}\right\rangle=\mathbf{S}_{i, 1}^{k} \mathbf{x}_{k-1, n}+\sum_{j=2}^{n} \mathbf{S}_{i, j}^{k} \mathbf{x}_{k, j}-\mathbf{Q}_{i, 1}^{k, p} \mathbf{x}_{k-r_{p}-2, n}-\sum_{p=1}^{v} \sum_{j=2}^{n} \mathbf{Q}_{i, j}^{k, p} \mathbf{x}_{k-r_{p}-1, j} \\
-\mathbf{R}_{i, 1}^{k, p} \mathbf{x}_{k-r_{p}-1, n}-\sum_{p=1}^{v} \sum_{j=2}^{n} \mathbf{R}_{i, j}^{k, p} \mathbf{x}_{k-r_{p}, j}=\mathbf{0}
\end{aligned}
$$

where

$$
\begin{aligned}
\mathbf{S}_{i, j}^{k} & =\int_{-1}^{1}\left(\frac{2}{h} \mathbf{I} \phi_{j}^{\prime}(\zeta)-\mathbf{A}\left(\frac{h(\zeta+1)}{2}+(k-1) h\right) \phi_{j}(\zeta)\right) \psi_{i}(\zeta) \mathrm{d} \zeta \\
\mathbf{Q}_{i, j}^{k, p} & =\int_{-1}^{-1+\beta_{p}} \mathbf{B}_{p}\left(\frac{h(\zeta+1)}{2}+(k-1) h\right) \phi_{j}\left(\zeta+2-\beta_{p}\right) \psi_{i}(\zeta) \mathrm{d} \zeta, \\
\mathbf{R}_{i, j}^{k, p} & =\int_{-1+\beta_{p}}^{1} \mathbf{B}_{p}\left(\frac{h(\zeta+1)}{2}+(k-1) h\right) \phi_{j}\left(\zeta-\beta_{p}\right) \psi_{i}(\zeta) \mathrm{d} \zeta .
\end{aligned}
$$

Note that the maximum degree of polynomials within these integral terms is $2 n-3$, that is, on $n$ number of $\eta_{q}, q=1,2, \ldots, n$ quadrature nodes, the Lobatto-type Gaussian quadrature (see 
Appendix C gives exact results for the integral terms by

$$
\begin{aligned}
\mathbf{S}_{i, j}^{k} & =\frac{2}{h} \mathbf{I} \sum_{q=1}^{n} \sum_{l=1}^{n} F_{i, q} L_{q, l} D_{l, j}-\sum_{q=1}^{n} F_{i, q} \mathbf{A}_{q}^{k} L_{q, j} \\
\mathbf{Q}_{i, j}^{k, p} & =\sum_{q=1}^{n} F_{i, q}^{Q, p} \mathbf{B}_{q}^{Q, k, p} L_{q, j}^{Q, p} \\
\mathbf{R}_{i, j}^{k, p} & =\sum_{q=1}^{n} F_{i, q}^{R, p} \mathbf{B}_{q}^{R, k, p} L_{q, j}^{R, p},
\end{aligned}
$$

where terms, corresponding to the periodic coefficients are calculated as

$$
\begin{aligned}
\mathbf{A}_{q}^{k} & =\mathbf{A}\left(\frac{h}{2}\left(\eta_{q}+1\right)+(k-1) h\right), \\
\mathbf{B}_{q}^{Q, k, p} & =\mathbf{B}_{p}\left(\frac{\alpha_{p}}{2}\left(\eta_{q}+1\right)+(k-1) h\right), \\
\mathbf{B}_{q}^{R, k, p} & =\mathbf{B}_{p}\left(\frac{h-\alpha_{p}}{2}\left(\eta_{q}+1\right)+\alpha_{p}+(k-1) h\right),
\end{aligned}
$$

while the terms containing $\psi_{i}$ test functions and $w_{q}$ quadrature weights are defined by

$$
\begin{aligned}
F_{i, q} & =\psi_{i}\left(\eta_{q}\right) w_{q}, \\
F_{i, q}^{Q, p} & =\frac{w_{q} \beta_{p}}{2} \psi_{i}\left(\frac{\beta_{p}}{2}\left(\eta_{q}+1\right)-1\right), \\
F_{i, q}^{R, p} & =\frac{w_{q}\left(2-\beta_{p}\right)}{2} \psi_{i}\left(\frac{\left(2-\beta_{p}\right) \eta_{q}+\beta_{p}}{2}\right),
\end{aligned}
$$

and the terms corresponding to $\phi_{j}$ Lagrange base polynomials are given by

$$
\begin{aligned}
L_{q, j} & =\phi_{j}\left(\eta_{q}\right), \\
L_{q, j}^{Q, p} & =\phi_{j}\left(\frac{\beta_{p}}{2}\left(\eta_{q}-1\right)+1\right), \\
L_{q, j}^{R, p} & =\phi_{j}\left(\frac{\left(2-\beta_{p}\right) \eta_{q}-\beta_{p}}{2}\right) .
\end{aligned}
$$

The derivative of a $\phi_{j}$ Lagrange base polynomial at a $\zeta_{l}$ node of interpolation is denoted by

$$
D_{l, j}=\phi_{j}^{\prime}\left(\zeta_{l}\right) \text {. }
$$

The barycentric formula

$$
\phi_{j}(\zeta)=\frac{\frac{\varpi_{j}}{\zeta-\zeta_{j}}}{\sum_{l=1}^{n} \frac{\varpi_{l}}{\zeta-\zeta_{l}}}
$$

of Lagrange base polynomials is more resistant to numerical instability than their ordinary form, hence here and in [20], (47) is applied. In (47), the so called barycentric weights are given by

$$
\varpi_{j}=\frac{1}{\omega^{\prime}\left(\zeta_{j}\right)}, \quad \omega(\zeta)=\prod_{j=1}^{n}\left(\zeta-\zeta_{j}\right) .
$$

The barycentric form 477) provides a compact formula

$$
\phi_{j}^{\prime}\left(\zeta_{l}\right)= \begin{cases}\frac{\varpi_{j} / \varpi_{l}}{\zeta_{l}-\zeta_{j}} & \text { if } j \neq l, \\ -\sum_{e \neq j}^{n} \frac{\varpi_{e} / \varpi_{j}}{\zeta_{j}-\zeta_{e}} & \text { if } j=l,\end{cases}
$$


for the derivatives of Lagrange base polynomials at the nodes of interpolation. For details on barycentric Lagrange interpolation see [6]. System (30) of algebraic equations, together with $\mathbf{x}_{0, n}=\mathbf{x}_{1,1}$, define the mapping

$$
\mathbf{H X}^{0, T}=\mathbf{G X}^{-\tau, 0},
$$

where $\mathbf{H}$ is a square matrix, while $\mathbf{X}^{0, T} \in \mathbb{R}^{s(E(n-1)+1) \times 1}, \mathbf{X}^{-\tau, 0} \in \mathbb{R}^{s(K E(n-1)+1) \times 1}$ and their elements are given by

$$
\mathbf{X}^{A h, B h}=\left\{\mathbf{X}_{u}^{A h, B h}\right\}_{u=1}^{(B-A)(n-1)+1}
$$

and

$$
\mathbf{X}_{u}^{A h, B h}=\mathbf{x}_{k, j}
$$

with

$$
\begin{aligned}
& k= \begin{cases}1+A & \text { if } u=1, \\
\operatorname{int}\left(\frac{u-2}{n-1}\right)+1+A & \text { otherwise, }\end{cases} \\
& j= \begin{cases}1 & \text { if } u=1, \\
u-(k-1-A)(n-1) & \text { otherwise. }\end{cases}
\end{aligned}
$$

Matrices $\mathbf{G}$ and $\mathbf{H}$ can be calculated according to

$$
\mathbf{G}=\sum_{p=0}^{v} \mathbf{G}_{p}, \quad \mathbf{H}=\sum_{p=0}^{v} \mathbf{H}_{p},
$$

where matrices $\mathbf{G}_{p}$ and $\mathbf{H}_{p}$ are constructed as shown in Figure 2, where the submatrices are defined according to

$$
\begin{aligned}
\mathbf{S}^{k} & =\left\{\mathbf{S}_{i, j}^{k}\right\}_{i, j=1}^{n-1, n} \in \mathbb{R}^{s(n-1) \times s n}, \quad k=1,2, \ldots, E ; \\
\mathbf{Q}^{k, p} & =\left\{\mathbf{Q}_{i, j}^{k, p}\right\}_{i, j=1}^{n-1, n} \in \mathbb{R}^{s(n-1) \times s n}, \quad k=1,2, \ldots, E ; \quad p=1,2, \ldots, v ; \\
\mathbf{R}^{k, p} & =\left\{\mathbf{R}_{i, j}^{k, p}\right\}_{i, j=1}^{n-1, n} \in \mathbb{R}^{s(n-1) \times s n}, \quad k=1,2, \ldots, E ; \quad p=1,2, \ldots, v .
\end{aligned}
$$

Note that matrices $\mathbf{Q}^{k, p}, k=1,2, \ldots, E$ are zero when $\tau_{p} \bmod h=0$. In Figure 2, the empty parts of matrices $\mathbf{G}_{p}$ and $\mathbf{H}_{p}$ are also zeros. The overlapping parts of submatrices $\mathbf{Q}^{k, p}$ and $\mathbf{R}^{k, p}$ are highlighted by circles. In these overlapping parts $s$ number of columns of $\mathbf{Q}^{k, p}$ and $\mathbf{R}^{k, p}$ overlap. The overlapping elements are merged by summation. Note, that matrix $\mathbf{S}^{k+1}$ is shifted to the right by $s(n-1)$ number of columns with respect to $\mathbf{S}^{k}$. Since the elements of $\mathbf{X}^{-\tau+T, T}$ and $\mathbf{X}^{-\tau, 0}$ precisely define the piecewise Lagrange interpolant of $\mathbf{x}^{-\tau+T, T}$ and $\mathbf{x}^{-\tau, 0}$, respectively, an approximation of the monodromy operator is given by the mapping

$$
\mathbf{X}^{-\tau+T, T}=\mathbf{U ~ X ~}^{-\tau, 0} .
$$

For the case $T<\tau_{v}$, the structure of $\mathbf{U}$ is shown in Figure 3 , where $\mathbf{I}$ and $\boldsymbol{\Theta}$ are identity and null matrices, respectively, depicted with their corresponding dimensions. For the case $T \geq \tau_{v}$, $K=1$ and $\tau=T$ due to (6), hence the identity block in $\mathbf{U}$ disappears. Consequently, the matrix approximation of the monodromy operator becomes simply

$$
\mathbf{U}=\mathbf{H}^{-1} \mathbf{G} \text {. }
$$

The stability of the approximate system (59) can be checked easily after the computation of the eigenvalues of $\mathbf{U}$, since (59) is stable if and only if all eigenvalues of $\mathbf{U}$ are located within the unit circle of the complex plane. In order to show the effectiveness of the method, in the next section, some results are presented for the stability charts of particular time-periodic DDEs. 

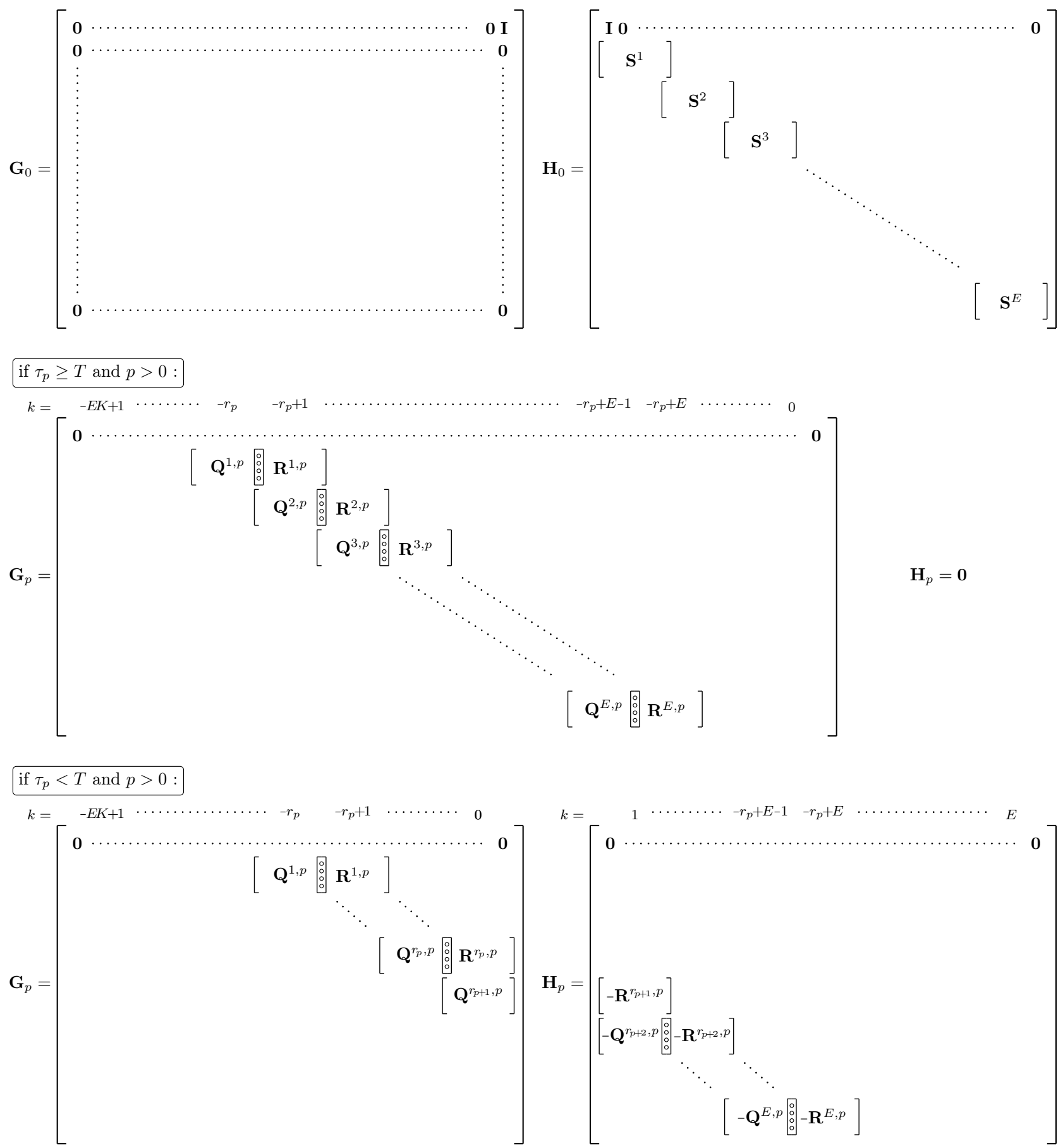

Figure 2: Structure of matrices $\mathbf{G}_{p}$ and $\mathbf{H}_{p}$ for $p=0,1, \ldots, v$. 


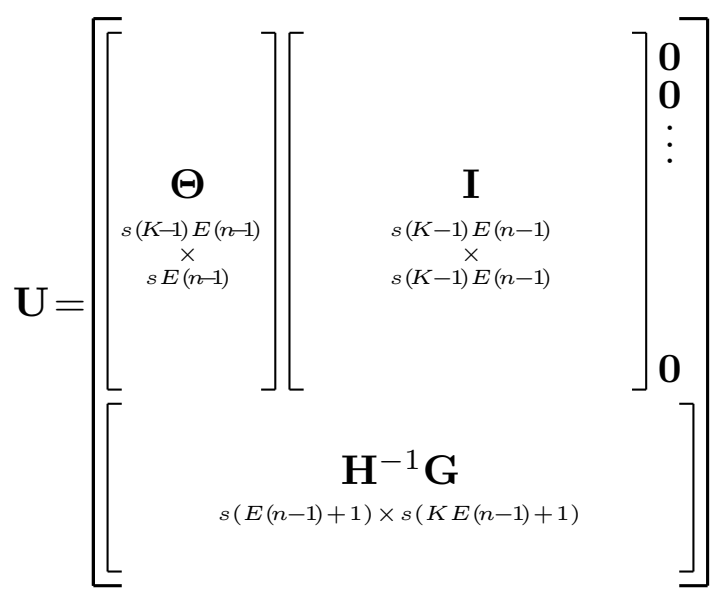

Figure 3: Structure of the matrix approximation $\mathbf{U}$ of monodromy operator $\mathcal{U}(T)$ in case $T<\tau_{v}$.

\section{Application}

In this section, results are presented for some particular time-periodic DDEs in the form of stability charts (see in Figures 4.9p computed by the spectral element method. First, the general settings of the computational method is described. Then, the particular DDEs are given and the computed results are discussed. Finally, computational details are shown for one of these examples under a particular set of computational and system parameters.

\subsection{General settings}

In [18, 19, 20], the nodes of interpolation are chosen to be the same as the quadrature nodes, that is the Lobatto-type Legendre nodes. The application of quadrature nodes for interpolation is beneficial, since this reduces (34) to

$$
\mathbf{S}_{i, j}^{k}=\frac{2}{h} \mathbf{I} \sum_{q=1}^{n} F_{i, q} D_{q, j}-F_{i, j} \mathbf{A}_{j}^{k} .
$$

The results in [18] show high convergence rates, therefore, in the following, the Lobatto-type Legendre nodes are used for interpolation. Note, however that the derived formulas enable the application of arbitrary Lobatto-type node sets. The same way as in [18], the test function set is chosen to be the set of Legendre polynomials up to degree $n-2$ (see Appendix B for details). For all the examples of the next subsection, only one element is used, that is $E=1$. The number $n$ of the nodes of interpolation and (if necessary) the number $m$ of quadrature nodes, used in (4), are given for each example (see the captions of Figures 44 9).

The stability charts are constructed in the plane of system parameters and are determined as follows. Eigenvalues of matrix $\mathbf{U}$ are computed on an equidistant $300 \times 300$ grid in the plane of system parameters. Eigenvalues having the largest absolute value are stored in each girdpoint. On these absolute values a 3-dimensional surface is fitted over the parameter plane using the "contour" function of Matlab. Thereafter, the approximate border of stability is obtained as a level curve of this 3 -dimensional surface at 1 . Note that the efficiency of this method can be increased if system parameters are computed sparsely far from the boundary of stability and with an increasing density close to the boundary of stability (for such methods see [7] and [4]). 


\subsection{Case studies}

The spectral element method is applied for six particular time-periodic DDEs (shown below) using the settings discussed in the previous subsection. For Examples 1 15, references are available in the literature, and the results can be directly compared. In case of Example 6. no results can be found in the literature, therefore, the results presented for this example were verified by numerical convergence analysis of the stability boundaries. This convergence analysis is not detailed here, however, the presented stability diagrams of Example 6 show the converged stability boundaries.

Example 1 (see Figure 4 and compare with the analytical results of [16])

$$
\ddot{x}(t)+(a+\varepsilon \cos (t)) x(t)=b x(t-2 \pi)
$$

Example 2 (see Figure 5 and compare with Fig. 11 in [10] and Fig. 7 in [8])

$$
\ddot{x}(t)+(a+\varepsilon \cos (t)) x(t)=b x(t-2 \pi)+\varepsilon x(t-4 \pi)
$$

Example 3 (see Figure 6 and compare with Fig. 6 in [8] and Fig. 10 in [20])

$$
\ddot{x}(t)+(6+\varepsilon \cos (2 \pi t)) x(t)=x\left(t-\tau_{1}\right)+x\left(t-\tau_{2}\right)
$$

Example 4 (see Figure 7 and compare with Fig. 4 in [8] and Fig. 4.9 in [17])

$$
\ddot{x}(t)+(a+\varepsilon \cos (4 \pi t)) x(t)=b \int_{-1}^{0} \frac{\pi}{2} \sin (\pi \theta) \mathbf{x}(t+\theta) \mathrm{d} \theta
$$

Example 5 (see Figure 8 and compare with Fig. 5 in [8] and Fig. 4.10 in [17])

$$
\ddot{x}(t)+(a+\varepsilon \cos (4 \pi t)) x(t)=b \int_{-1}^{0}\left(\frac{\pi}{2} \sin (\pi \theta)+\frac{13 \pi}{77} \sin (2 \pi \theta)\right) \mathbf{x}(t+\theta) \mathrm{d} \theta
$$

Example 6 (see Figure 9)

$$
\ddot{x}(t)+(a+\varepsilon \cos (4 \pi t)) x(t)=b \cos (\pi t) \int_{-1}^{0} \frac{\pi}{2} \sin (\pi \theta) \mathbf{x}(t+\theta) \mathrm{d} \theta
$$

It can be seen in Figures 48 , that the stability charts are the same as those given in the corresponding references. Note for these examples that the number $n$ of interpolation nodes is smaller than or equal to the approximation number of the results presented in the corresponding references (when numerical results are given in the reference). This implies that the spectral element method is competitive with the methods available in the literature. Note however that for precise comparison the time necessary for computation is of interest rather than the approximation number. 

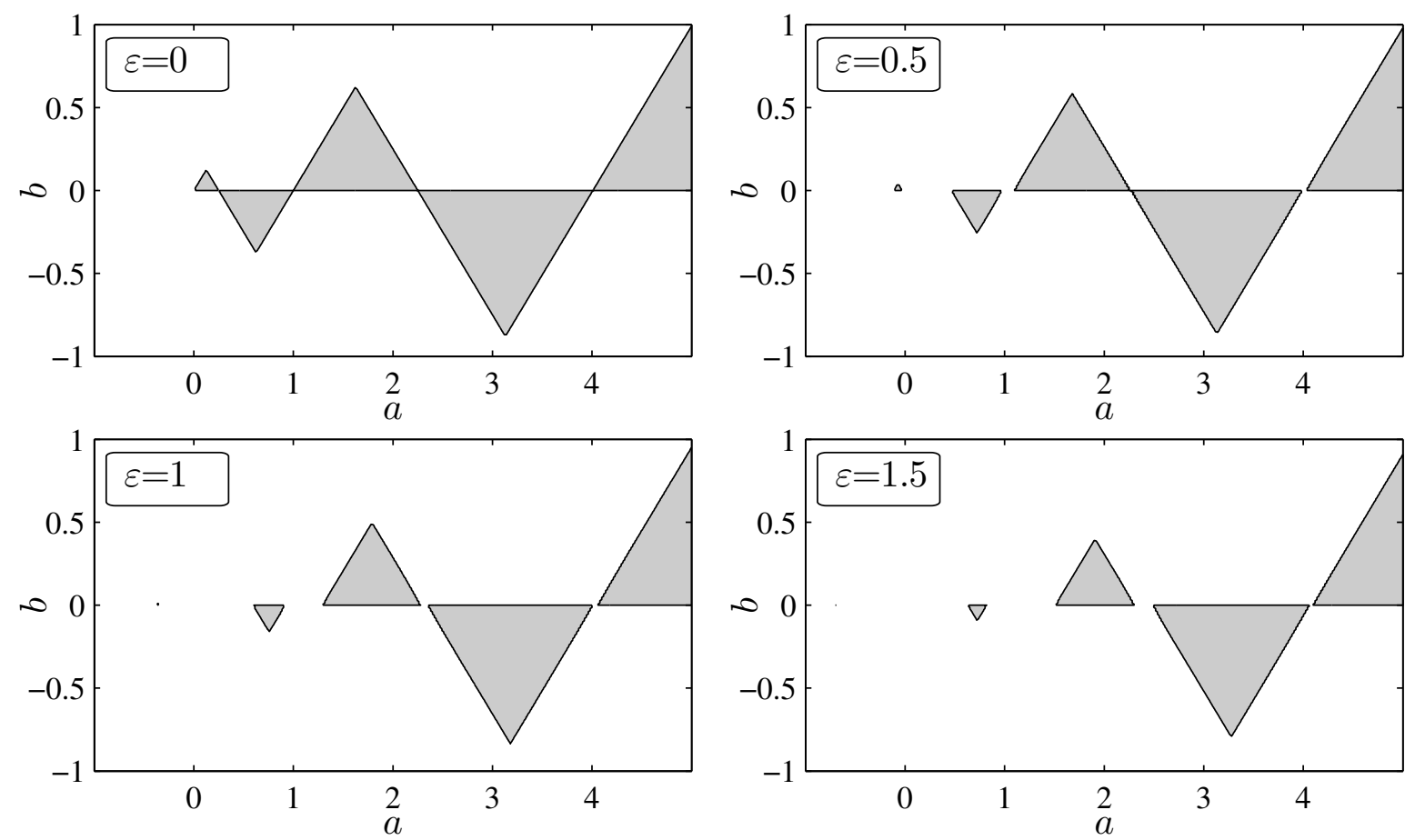

Figure 4: Stability charts of Example 1 for different $\varepsilon$ values. The number of elements is $E=1$, the number of interpolation nodes is $n=10$.
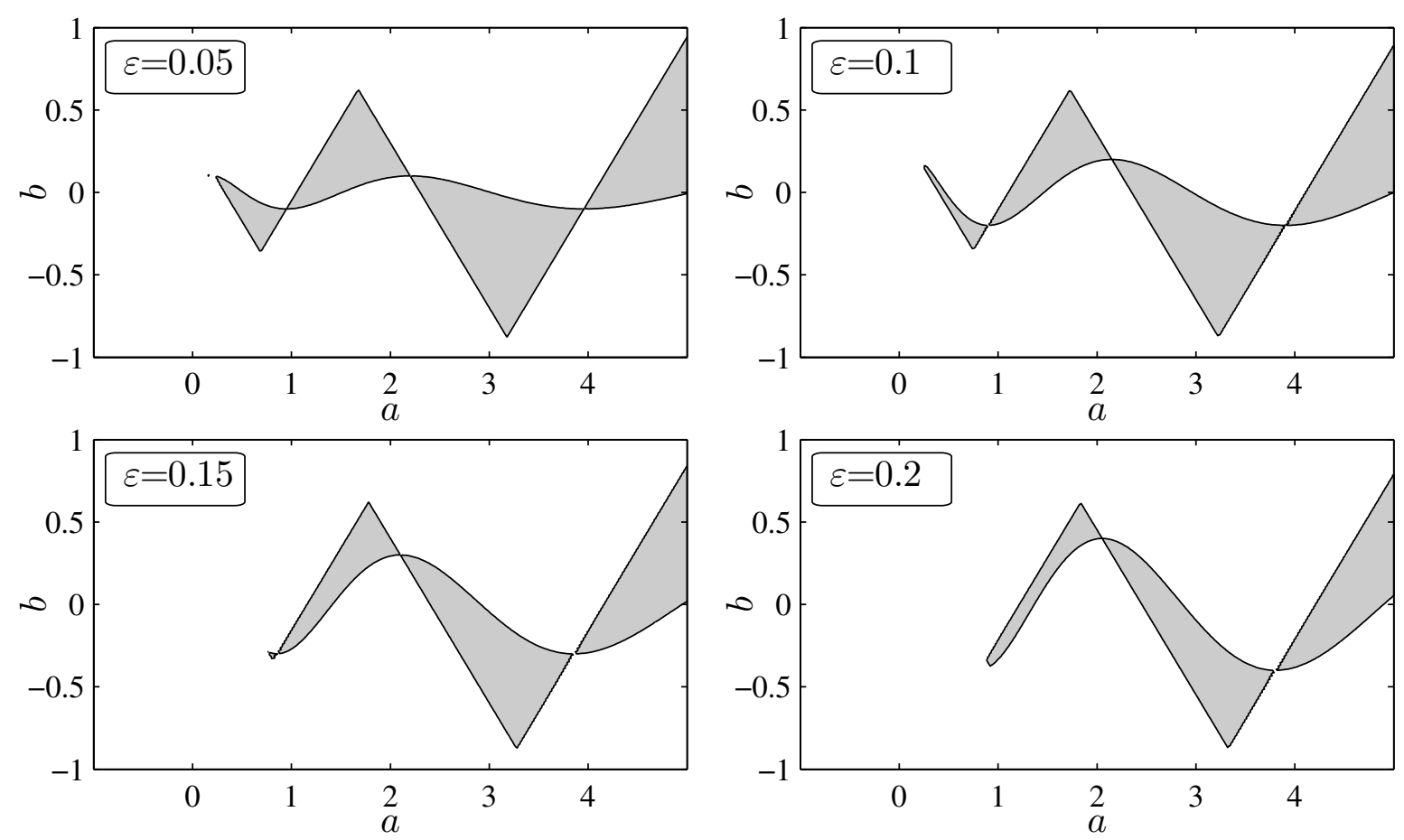

Figure 5: Stability charts of Example 2 for different $\varepsilon$ values. The number of elements is $E=1$, the number of interpolation nodes is $n=10$. 

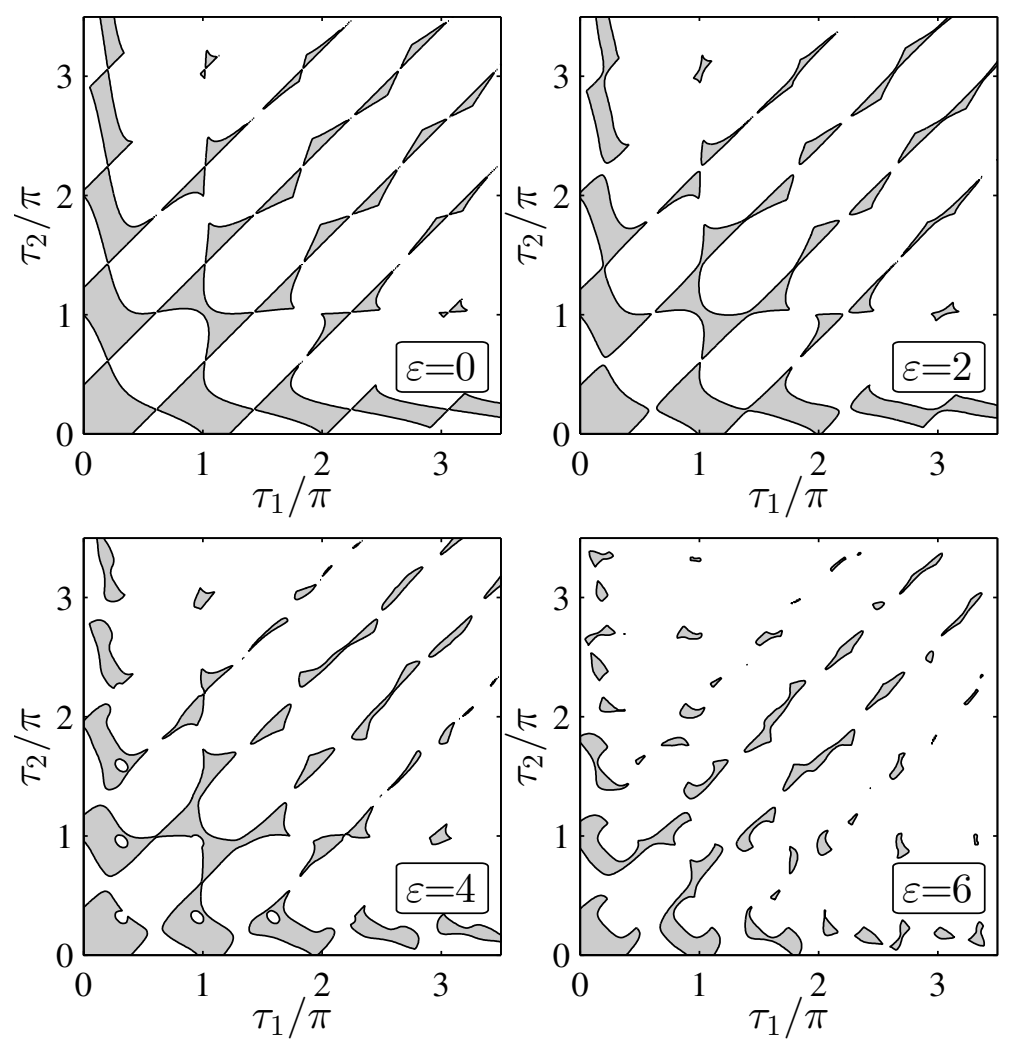

Figure 6: Stability charts of Example 3 for different $\varepsilon$ values. The number of elements is $E=1$, the number of interpolation nodes is $n=10$.

\subsection{Numerical example}

Section 2 provides compact formulas for the construction of the matrix approximation $\mathbf{U}$ of the monodromy operator for the general case (4). However, application of these formulas to specific examples is not always straightforward. In order to help to understand the process of computation, a computational example is given in this subsection for Example 3, under particular computational and system parameters. In addition, a Matlab code is also provided for the calculation of stability charts corresponding to Example 6 through the link: http: //www.mm.bme.hu/ lehotzky/Case_5.m.

The first-order form of (64) reads

$$
\dot{\mathbf{x}}(t)=\mathbf{A}(t) \mathbf{x}(t)+\mathbf{B} \mathbf{x}\left(t-\tau_{1}\right)+\mathbf{B} \mathbf{x}\left(t-\tau_{2}\right),
$$

where

$$
\mathbf{x}(t)=\left[\begin{array}{c}
x(t) \\
\dot{x}(t)
\end{array}\right], \mathbf{A}(t)=\left[\begin{array}{cc}
0 & 1 \\
-6-\varepsilon \cos (2 \pi t) & 0
\end{array}\right], \mathbf{B}=\left[\begin{array}{ll}
0 & 0 \\
1 & 0
\end{array}\right] .
$$

Assume that $E=3$ and $n=3$, that is 3 elements and 3 interpolation nodes are used. Consequently, the length of the elements is $h=T / 3$ and the Lobatto-type Gaussian quadrature nodes and weights are $\eta_{1}=-1, \eta_{2}=0, \eta_{3}=1$ and $w_{1}=1 / 3, w_{2}=4 / 3, w_{3}=1 / 3$; respectively (see Appendix C . Note that the nodes of interpolation are the same as the quadrature nodes, that is $\zeta_{j}=\eta_{j}$ for $j=1,2,3$. The test functions are the Legendre polynomials up to degree 1 , hence $\psi_{1}(\zeta)=1$ and $\psi_{2}(\zeta)=\zeta$ (see Appendix B). Assume that $2 h<\tau_{1}<3 h$ and $4 h<\tau_{2}<5 h$, therefore (6) gives $K=2$, which results in $r_{1}=2$ and $r_{2}=4$. After $\alpha_{1}=\tau_{1} \bmod h, \alpha_{2}=\tau_{2} \bmod h$ and $\beta_{1}=2 \alpha_{1} / h, \beta_{2}=2 \alpha_{2} / h$ are computed, the submatrices (34)-(36) can be calculated using formulas (37)-(49). Using submatrices (34)-(36), matrices $\mathbf{H} \in \mathbb{R}^{14 \times 14}$ and $\mathbf{G} \in \mathbb{R}^{14 \times 26}$ can be 

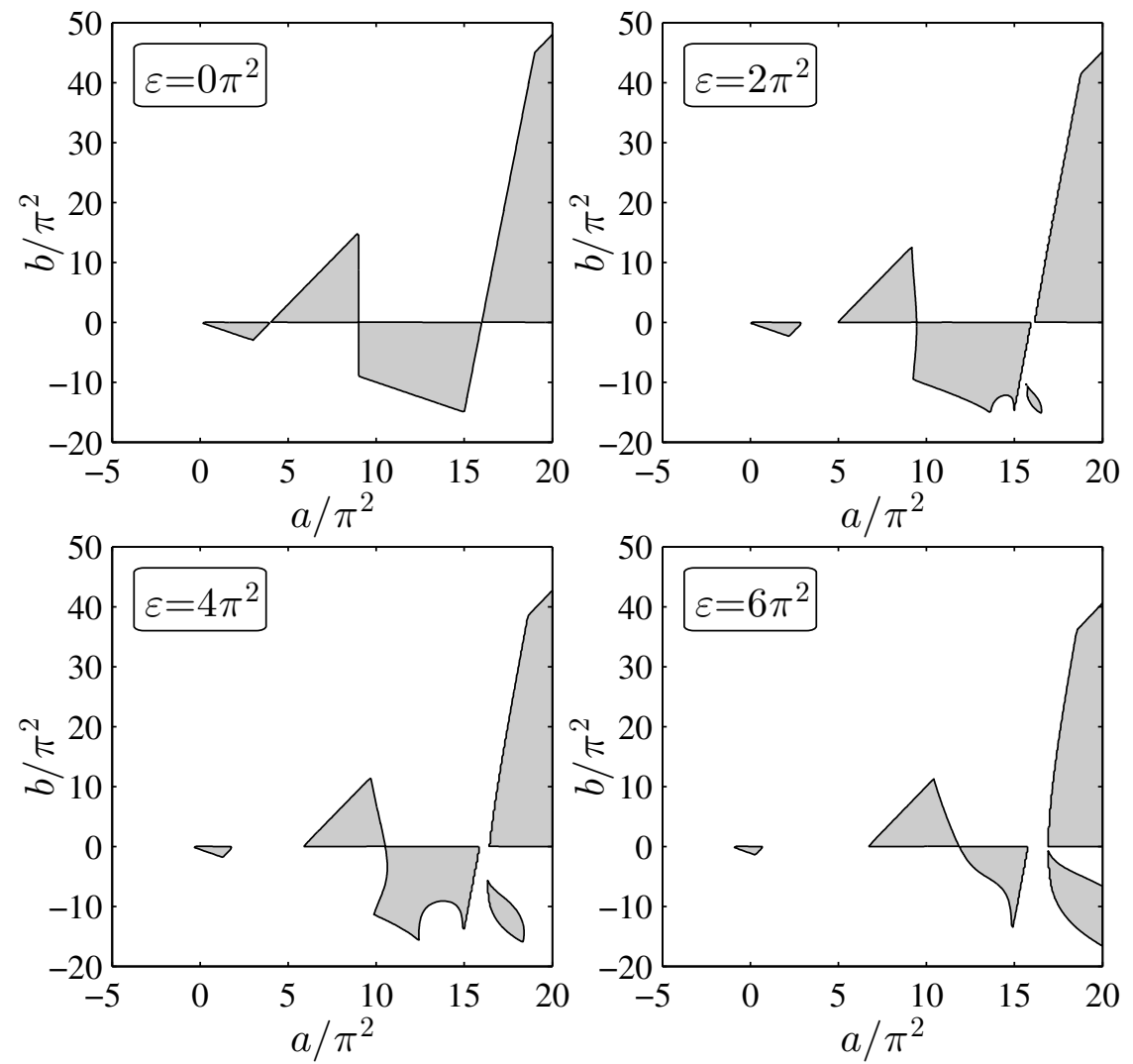

Figure 7: Stability charts of Example 4 for different $\varepsilon$ values. The number of elements is $E=1$, the

number of interpolation nodes is $n=10$ and the number of quadrature nodes in (4) is $m=10$. 

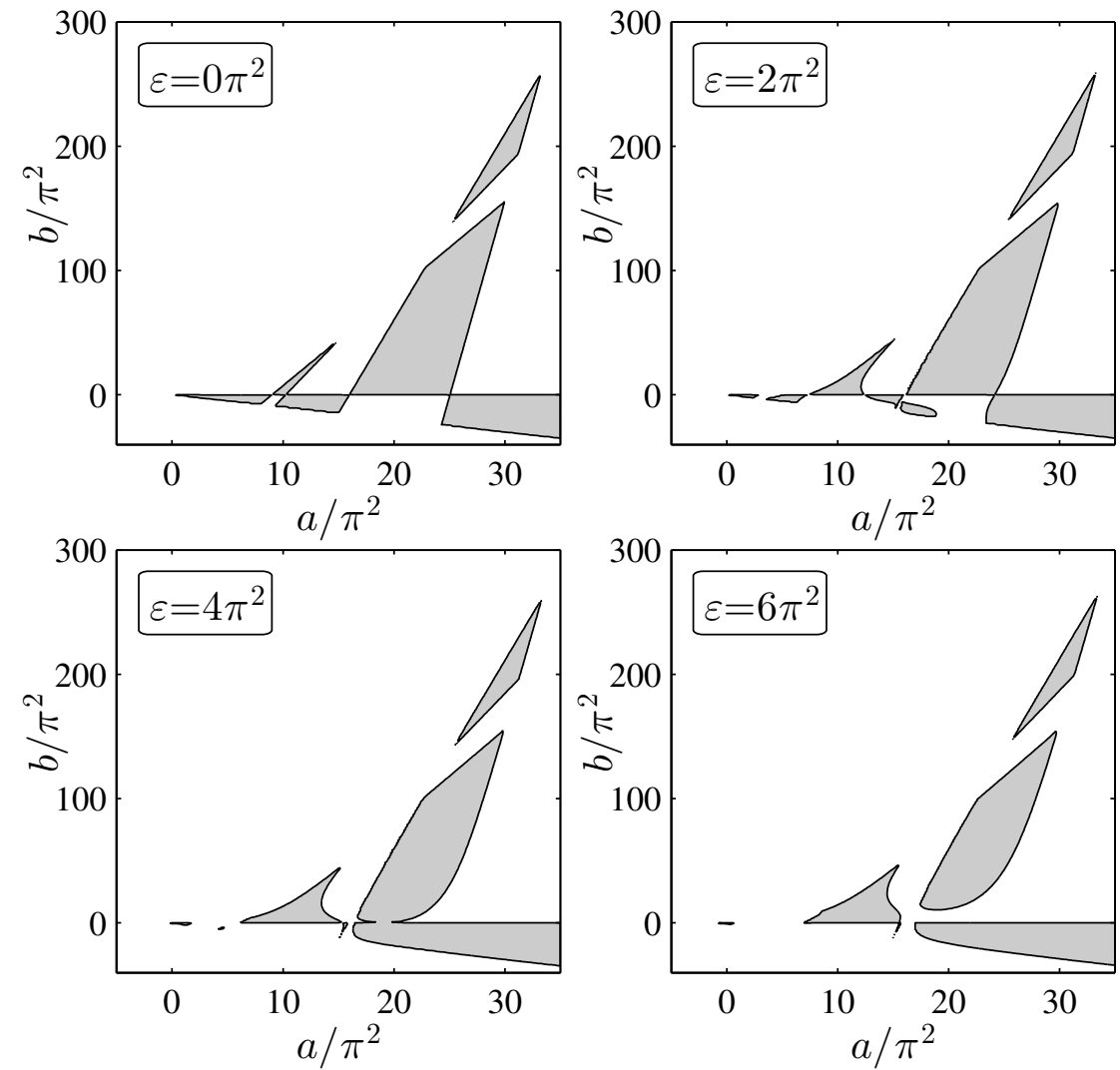

Figure 8: Stability charts of Example 5 for different $\varepsilon$ values. The number of elements is $E=1$, the number of interpolation nodes is $n=10$ and the number of quadrature nodes in (4) is $m=10$. 

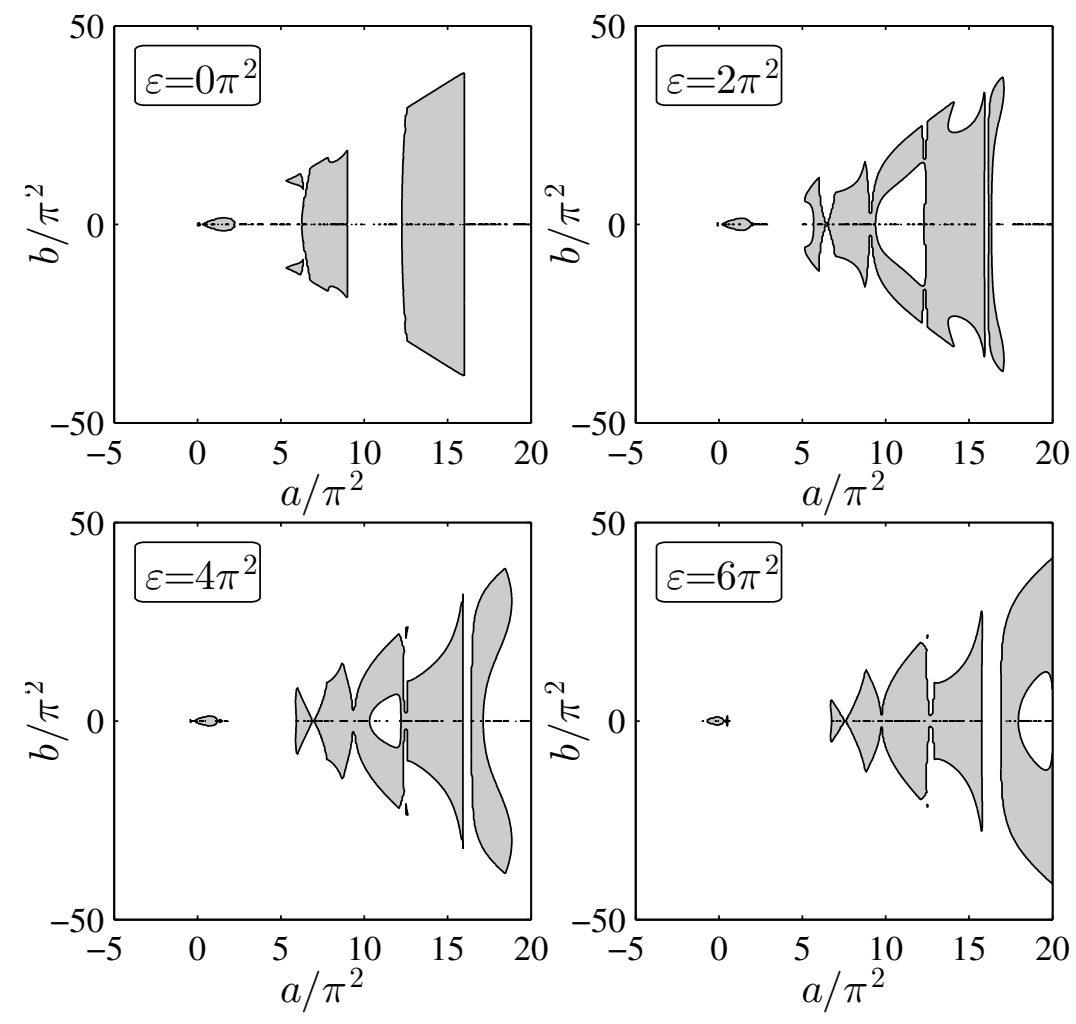

Figure 9: Stability charts of Example 6 for different $\varepsilon$ values. The number of elements is $E=1$, the number of interpolation nodes is $n=40$ and the number of quadrature nodes in (4) is $m=40$.

computed from (55). The matrices $\mathbf{H}_{0}$ and $\mathbf{H}_{1}$ in (55) are given by (70), while $\mathbf{H}_{2}=\mathbf{0}$ because $\tau_{2}>T$. The structure of $\mathbf{G}_{0}$ is shown in Figure 2, while matrices $\mathbf{G}_{1}$ and $\mathbf{G}_{2}$ are given in (71) and $(72)$, respectively. Knowing that $\tau_{1}<T$ and $\tau_{2}>T$, the structure of $\mathbf{G}_{1}$ and $\mathbf{G}_{2}$ can be verified by the corresponding matrix structures shown in Figure 2, The matrix approximation $\mathbf{U} \in \mathbb{R}^{26 \times 26}$ of the monodromy operator can be constructed according to Figure 3 , using (50).

$$
\begin{gathered}
\mathbf{H}_{0}=\left[\begin{array}{ccccccc}
\mathbf{I} & \mathbf{0} & \mathbf{0} & \mathbf{0} & \mathbf{0} & \mathbf{0} & \mathbf{0} \\
\mathbf{S}_{1,1}^{1} & \mathbf{S}_{1,2}^{1} & \mathbf{S}_{1,3}^{1} & \mathbf{0} & \mathbf{0} & \mathbf{0} & \mathbf{0} \\
\mathbf{S}_{2,1}^{1} & \mathbf{S}_{2,2}^{1} & \mathbf{S}_{2,3}^{1,} & \mathbf{0} & \mathbf{0} & \mathbf{0} & \mathbf{0} \\
\mathbf{0} & \mathbf{0} & \mathbf{S}_{1,1}^{2} & \mathbf{S}_{1,2}^{2} & \mathbf{S}_{1,3}^{2} & \mathbf{0} & \mathbf{0} \\
\mathbf{0} & \mathbf{0} & \mathbf{S}_{2,1}^{2} & \mathbf{S}_{2,2}^{2} & \mathbf{S}_{2,3}^{2} & \mathbf{0} & \mathbf{0} \\
\mathbf{0} & \mathbf{0} & \mathbf{0} & \mathbf{0} & \mathbf{S}_{1,1}^{3} & \mathbf{S}_{1,2}^{3} & \mathbf{S}_{1,3}^{3} \\
\mathbf{0} & \mathbf{0} & \mathbf{0} & \mathbf{0} & \mathbf{S}_{2,1}^{3} & \mathbf{S}_{2,2}^{3,} & \mathbf{S}_{2,3}^{3}
\end{array}\right], \mathbf{H}_{1}=\left[\begin{array}{ccccccc}
\mathbf{0} & \mathbf{0} & \mathbf{0} & \mathbf{0} & \mathbf{0} & \mathbf{0} & \mathbf{0} \\
\mathbf{0} & \mathbf{0} & \mathbf{0} & \mathbf{0} & \mathbf{0} & \mathbf{0} & \mathbf{0} \\
\mathbf{0} & \mathbf{0} & \mathbf{0} & \mathbf{0} & \mathbf{0} & \mathbf{0} & \mathbf{0} \\
\mathbf{0} & \mathbf{0} & \mathbf{0} & \mathbf{0} & \mathbf{0} & \mathbf{0} & \mathbf{0} \\
\mathbf{0} & \mathbf{0} & \mathbf{0} & \mathbf{0} & \mathbf{0} & \mathbf{0} & \mathbf{0} \\
-\mathbf{R}_{1,1}^{3,1} & -\mathbf{R}_{1,2}^{3,1} & -\mathbf{R}_{1,3}^{3,1} & \mathbf{0} & \mathbf{0} & \mathbf{0} & \mathbf{0} \\
-\mathbf{R}_{2,1}^{3,1} & -\mathbf{R}_{2,2}^{3,1} & -\mathbf{R}_{2,3}^{3,1} & \mathbf{0} & \mathbf{0} & \mathbf{0} & \mathbf{0}
\end{array}\right] \\
\mathbf{G}_{1}= \\
\left.\begin{array}{llllllllllllllll}
\mathbf{0} & \mathbf{0} & \mathbf{0} & \mathbf{0} & \mathbf{0} & \mathbf{0} & \mathbf{0} & \mathbf{0} & \mathbf{0} & \mathbf{0} & \mathbf{0} & \mathbf{0} & \mathbf{0} \\
\mathbf{0} & \mathbf{0} & \mathbf{0} & \mathbf{0} & \mathbf{0} & \mathbf{0} & \mathbf{Q}_{1,1}^{1,1} & \mathbf{Q}_{1,2}^{1,1} & \mathbf{Q}_{1,3}^{1,1}+\mathbf{R}_{1,1}^{1,1} & \mathbf{R}_{1,2}^{1,1} & \mathbf{R}_{1,3}^{1,1} & \mathbf{0} & \mathbf{0} \\
\mathbf{0} & \mathbf{0} & \mathbf{0} & \mathbf{0} & \mathbf{0} & \mathbf{0} & \mathbf{Q}_{2,1}^{1,1} & \mathbf{Q}_{2,2}^{1,1} & \mathbf{Q}_{2,3}^{1,1}+\mathbf{R}_{2,1}^{1,1} & \mathbf{R}_{2,2}^{1,1} & \mathbf{R}_{2,1}^{1,1} & \mathbf{0} & \mathbf{0} \\
\mathbf{0} & \mathbf{0} & \mathbf{0} & \mathbf{0} & \mathbf{0} & \mathbf{0} & \mathbf{0} & \mathbf{0} & \mathbf{Q}_{1,1}^{2,1} & \mathbf{Q}_{1,2}^{2,1} & \mathbf{Q}_{1,3}^{2,1}+\mathbf{R}_{1,1}^{2,1} & \mathbf{R}_{1,2}^{2,1} & \mathbf{R}_{1,3}^{2,1} \\
\mathbf{0} & \mathbf{0} & \mathbf{0} & \mathbf{0} & \mathbf{0} & \mathbf{0} & \mathbf{0} & \mathbf{0} & \mathbf{Q}_{2,1}^{2,1} & \mathbf{Q}_{2,1}^{2,1} & \mathbf{Q}_{2,3}^{2,1}+\mathbf{R}_{2,1}^{2,1} & \mathbf{R}_{2,2}^{2,1} & \mathbf{R}_{2,3}^{2,1} \\
\mathbf{0} & \mathbf{0} & \mathbf{0} & \mathbf{0} & \mathbf{0} & \mathbf{0} & \mathbf{0} & \mathbf{0} & \mathbf{0} & \mathbf{0} & \mathbf{Q}_{1,1}^{3,1} & \mathbf{Q}_{1,2}^{3,1} & \mathbf{Q}_{1,3}^{3,1} \\
\mathbf{0} & \mathbf{0} & \mathbf{0} & \mathbf{0} & \mathbf{0} & \mathbf{0} & \mathbf{0} & \mathbf{0} & \mathbf{0} & \mathbf{0} & \mathbf{Q}_{2,1}^{3,1} & \mathbf{Q}_{2,2}^{3,1} & \mathbf{Q}_{2,3}^{3,1}
\end{array}\right]
\end{gathered}
$$




$$
\mathbf{G}_{2}=\left[\begin{array}{ccccccccccccc}
\mathbf{0} & \mathbf{0} & \mathbf{0} & \mathbf{0} & \mathbf{0} & \mathbf{0} & \mathbf{0} & \mathbf{0} & \mathbf{0} & \mathbf{0} & \mathbf{0} & \mathbf{0} & \mathbf{0} \\
\mathbf{0} & \mathbf{0} & \mathbf{Q}_{1,1}^{1,2} & \mathbf{Q}_{1,2}^{1,2} & \mathbf{Q}_{1,3}^{1,2}+\mathbf{R}_{1,1}^{1,2} & \mathbf{R}_{1,2}^{1,2} & \mathbf{R}_{1,3}^{1,2} & \mathbf{0} & \mathbf{0} & \mathbf{0} & \mathbf{0} & \mathbf{0} & \mathbf{0} \\
\mathbf{0} & \mathbf{0} & \mathbf{Q}_{2,1}^{1,2} & \mathbf{Q}_{2,2}^{1,2} & \mathbf{Q}_{2,3}^{1,2}+\mathbf{R}_{2,1}^{1,2} & \mathbf{R}_{2,2}^{1,2} & \mathbf{R}_{2,2}^{1,2} & \mathbf{0} & \mathbf{0} & \mathbf{0} & \mathbf{0} & \mathbf{0} & \mathbf{0} \\
\mathbf{0} & \mathbf{0} & \mathbf{0} & \mathbf{0} & \mathbf{Q}_{1,1}^{2,2} & \mathbf{Q}_{1,2}^{2,2} & \mathbf{Q}_{1,3}^{2,2}+\mathbf{R}_{1,1}^{2,2} & \mathbf{R}_{1,2}^{2,2} & \mathbf{R}_{1,3}^{2,2} & \mathbf{0} & \mathbf{0} & \mathbf{0} & \mathbf{0} \\
\mathbf{0} & \mathbf{0} & \mathbf{0} & \mathbf{0} & \mathrm{Q}_{2,1}^{2,2} & \mathbf{Q}_{2,2}^{2,2} & \mathbf{Q}_{2,3}^{2,2}+\mathbf{R}_{2,1}^{2,2} & \mathbf{R}_{2,2}^{2,2} & \mathbf{R}_{2,2}^{2,2} & \mathbf{0} & \mathbf{0} & \mathbf{0} & \mathbf{0} \\
\mathbf{0} & \mathbf{0} & \mathbf{0} & \mathbf{0} & \mathbf{0} & \mathbf{0} & \mathbf{Q}_{1,1}^{3,2} & \mathbf{Q}_{1,2}^{3,2} & \mathbf{Q}_{1,3}^{3,2}+\mathbf{R}_{1,1}^{3,2} & \mathbf{R}_{1,2}^{3,2} & \mathbf{R}_{1,3}^{3,2} & \mathbf{0} & \mathbf{0} \\
\mathbf{0} & \mathbf{0} & \mathbf{0} & \mathbf{0} & \mathbf{0} & \mathbf{0} & \mathbf{Q}_{2,1}^{3,2} & \mathbf{Q}_{2,2}^{3,2} & \mathbf{Q}_{2,3}^{3,2}+\mathbf{R}_{2,1}^{3,2} & \mathbf{R}_{2,2}^{3,2} & \mathbf{R}_{2,3}^{3,2} & \mathbf{0} & \mathbf{0}
\end{array}\right]
$$

Note that $\mathbf{B}_{q}^{Q, k, p}=\mathbf{B}_{q}^{R, k, p}=\mathbf{B}$ for $p=1,2 ; k=1,2,3$ and $q=1,2,3$, hence $\mathbf{Q}_{i, j}^{k, p}=\mathbf{Q}_{i, j}^{k+1, p}$ and $\mathbf{R}_{i, j}^{k, p}=\mathbf{R}_{i, j}^{k+1, p}$ for $k=1,2$. In order to determine stability boundaries, the matrix approximation $\mathbf{U}$ of the monodromy operator has to be computed in several points of the $\left(\tau_{1}, \tau_{2}\right)$ parameter plane. Note, however, that if $\tau_{1}$ and $\tau_{2}$ are changed, then only the terms $F_{i, q}^{Q, p}, F_{i, q}^{R, p}$ and $L_{i, q}^{Q, p}$, $L_{i, q}^{R, p}$ for $p=1,2 ; i=1,2$ and $q=1,2,3$ have to be recomputed and all the other terms in (37) $-(39)$ and (46)-(49) remain the same. This feature is reflected in the low time-demand of the calculation of stability charts.

\section{Conclusions}

A generalization of the spectral element method, introduced in [20], was presented for timeperiodic DDEs with point delays and distributed delay. An explicit formula was given for the construction of the matrix approximation of the monodromy operator. This formula enables the application of arbitrary point sets and test functions for general combinations of delays without any insight into the derivations of the method. Stability analysis was demonstrated for some case studies, and the computation code is also provided for the most general example.

\section{Acknowledgements}

This work was partially supported by the Hungarian National Science Foundation under grant OTKAK105433 and by the Hungarian-Chinese Bilateral Fund for Science and Technology under grant number TET_12_CN-1-2012-0012. The research leading to these results has received funding from the European Research Council under the European Unions Seventh Framework Programme (FP/2007-2013) / ERC Advanced Grant Agreement n. 340889. 


\section{Appendices}

\section{Appendix A Lagrange interpolation}

On a $t \in[a, b]$ interval the $\tilde{x}(t)$ Lagrange interpolant of an $x(t)$ function is an $(n-1)$-order polynomial satisfying conditions

$$
\tilde{x}\left(t_{j}\right)=x\left(t_{j}\right), \quad t_{j} \in[a, b], \quad j=1,2, \ldots, n
$$

where $t_{j}, j=1,2, \ldots, n$; are called the nodes of interpolation. The unique solution for the interpolant is

$$
\tilde{x}(t)=\sum_{j=1}^{n} \phi_{j}(t) \tilde{x}\left(t_{j}\right),
$$

where the Lagrange base polynomials are computed as

$$
\phi_{j}(t)=\prod_{\substack{k=1 \\ k \neq j}}^{n} \frac{t-t_{k}}{t_{j}-t_{k}}
$$

and have the property

$$
\phi_{j}\left(t_{k}\right)=\delta_{j, k}
$$

with $\delta_{j, k}$ being the Kronecker delta function.

\section{Appendix B Legendre polynomials}

Definition of Legendre polynomials by Bonnet's recursion formula:

$$
\begin{aligned}
& P_{1}=1, \\
& P_{2}=\zeta, \\
& P_{j}(\zeta)=\frac{2 j-3}{j-1} \zeta P_{j-1}(\zeta)-\frac{j-2}{j-1} P_{j-2}(\zeta) \quad j=3,4, \ldots
\end{aligned}
$$

Some properties of Legendre polynomials are

- orthogonality: $\int_{-1}^{1} P_{j}(\zeta) P_{i}(\zeta) \mathrm{d} \zeta=\frac{2}{2 j-1} \delta_{i, j}$ and

- $P_{j}(1)=1$ and $P_{j}(-1)=(-1)^{j-1}$.

\section{Appendix C Lobatto-type Gaussian quadrature}

The Lobatto-type Gaussian quadrature approximates a definite integral by a sum as

$$
I=\int_{a}^{b} x(t) \mathrm{d} t \approx \tilde{I}=\sum_{q=1}^{n} x\left(t_{q}\right) w_{q},
$$

where $t_{q}=\frac{a-b}{2} \eta_{q}+\frac{a+b}{2}$ with $\eta_{q}$ and $w_{q}$ being the quadrature nodes and weights, respectively. The Lobatto-type Gaussian quadrature gives exact results for all polynomials with maximum order $2 n-3$. The quadrature nodes are the roots of $\left(1-\zeta^{2}\right) P_{n}^{\prime}(\zeta)$, that is $-1,1$ and the roots of the first derivative of the Legendre polynomial with degree $(n-1)$. The quadrature weights are given by

$$
w_{q}= \begin{cases}\frac{2}{n(n-1)} & \text { if } q=1, n \\ \frac{2}{n(n-1) P_{n}^{2}\left(\eta_{q}\right)} & \text { if } q=2,3, \ldots, n-1 .\end{cases}
$$




\section{References}

[1] Altintas Y, Budak E. Analytical prediction of stability lobes in milling. CIRP Ann - Manuf Techn 1995;44:357-362.

[2] Altintas Y. Manufacturing Automation. 2nd ed. New York: Cambridge University Press; 2012 .

[3] Åström KJ, Murray RM. Feedback Systems. New Jersey: Princeton University Press; 2008.

[4] Bachrathy D, Stepan G. Bisection method in higher dimensions and the efficiency number. Period Polytech Mech 2012;56(2):81-86.

[5] Bachrathy D, Stepan G. Improved prediction of stability lobes with extended multi frequency solution. CIRP Ann - Manuf Techn 2013;62:411-414. http://dx.doi.org/10.1016/j.cirp.2013.03.085 .

[6] Berrut JP, Trefethen LN. Barycentric Lagrange Interpolation. SIAM Review 2004;46(3):501-517. http://eprints.maths.ox.ac.uk/1212/ .

[7] Breda D, Maset S, Vermiglio R. An adaptive algorithm for efficient computation of level curves of surfaces. Numer Algorithms 2009;52:605-628.

[8] Breda D, Maset S, Vermiglio R. Pseudospectral methods for stability analysis of delayed dynamical systems. Int J Dynam Control 2014;2:143-153. http://link.springer.com/10.1007/s40435-013-0041-x

[9] Sinha SC, Butcher EA. Symbolic computation of fundamental solution matrices for linear time-periodic dynamical systems. J Sound Vib 1997;206(1):61-85.

[10] Butcher EA, Bobrenkov OA. On the Chebyshev spectral continuous time approximation for constant and periodic delay differential equations. Commun Nonlinear Sci Numer Simul 2011,16:1541-1554. http://dx.doi.org/10.1016/j.cnsns.2010.05.037 .

[11] Crandall SH. Engineering analysis: a survey of numerical procedures. New York: McGrawHill; 1956.

[12] Ding Y, Zhu LM, Zhang XJ, Ding H. A full-discretization method for prediction of milling stability. Int J Mach Tools Manuf 2010;50:502-509.

[13] Habib G, Rega G, Stepan G. Delayed digital position control of a single-DoF system and the nonlinear behavior of the act-and-wait controller. J Vibr Control 2014;DOI:10.1177/1077546314533583.

[14] Hale JK, Lunel SMV. Introduction to functional differential equations. 1st ed. New York: Springer-Verlag; 1993.

[15] Insperger T, Milton J. Sensory uncertainty and stick balancing at the fingertip. Biol Cybern 2014;108:85-101.

[16] Insperger T, Stepan G. Stability chart for the delayed Mathieu equation. Proc R Soc Lond A-Math Phy 2002;458:1989-1998.

[17] Insperger T, Stepan G. Semi-discretization for time-delay systems. New York: Springer; 2011. 
[18] Khasawneh FA, Mann BP. A spectral element approach for the stability of delay systems. Int J Numer Meth Engng 2011;87:566-592.

[19] Khasawneh FA, Mann BP. Stability of delay integro-differential equations using a spectral element method. Math Computer Modelling 2011;54:2493-2503. http://dx.doi.org/10.1016/j.mcm.2011.06.009 .

[20] Khasawneh FA, Mann BP. A spectral element approach for the stability analysis of time-periodic delay equations with multiple delays. Commun Nonlinear Sci Numer Simul 2013;18:2129-2141. http://dx.doi.org/10.1016/j.cnsns.2012.11.030 .

[21] Kuang Y. Delay differential equations with applications in population dynamics. New York: Academic Press; 1993.

[22] Li M, Zhang G, Huang Y. Complete discretization scheme for milling stability prediction. Nonlinear Dyn 2013;71:187-199.

[23] Milton J, Cabrera JL, Ohira T, Tajima S, Tonosaki Y, Eurich CW, Campbell SA. The time-delayed inverted pendulum: Implications for human balance control. Chaos 2009;19:026110.

[24] Orosz G, Wilson RE, Szalai R, Stepan G. Exciting traffic jams: nonlinear phenomena behind traffic jam formation on highways. Phys Review E 2009;80(4):046205.

[25] Röst G, Wu J. SEIR epidemiological model with varying infectivity and infinite delay. Math Biosci Engng 2008;5(2):389-402.

[26] Szekrenyes A. A special case of parametrically excited systems: free vibration of delaminated composite beams. Eur J Mech A/Solids 2015;49:82-105.

[27] Stepan G, Munoa J, Insperger T, Surico M, Bachrathy D, Dombovari Z. Cylindrical milling tools: Comparative real case study for process stability. CIRP Ann - Manuf Techn 2014;63:385-388.

[28] Suzuki Y, Nomura T, Casadio M, Morasso P. Intermittent control with ankle, hip, and mixed strategies during quiet standing: A theoretical proposal based on a double inverted pendulum model. J Theor Biology 2012;310:55-79. http://dx.doi.org/10.1016/j.jtbi.2012.06.019 .

[29] Takacs D, Stepan G. Contact patch memory of tyres leading to lateral vibrations of fourwheeled vehicles. Phil Trans R Soc A 2013;371:20120427.

[30] Trefethen L N. Is Gauss Quadrature Better than Clenshaw-Curtis? SIAM Review 2008;50(1):67-87.

[31] Tweten DJ, Mann BP. Spectral element method and the delayed feedback control of chaos. Phys Rew E 2012;86:046214.

[32] Tweten DJ, Mann BP. Delayed feedback control of chaos for arbitrary delays with the spectral element method. Int J Dynam Control 2013;1:283-289. 